University of Windsor

Scholarship at UWindsor

Physics Publications

Department of Physics

2000

\title{
Photodetachment in combined static and dynamic electric fields
}

\author{
Chitra Rangan \\ University of Windsor
}

A.R.P. Rau

Follow this and additional works at: https://scholar.uwindsor.ca/physicspub

Part of the Physics Commons

\section{Recommended Citation}

Rangan, Chitra and Rau, A.R.P.. (2000). Photodetachment in combined static and dynamic electric fields. Physical Review A - Atomic, Molecular, and Optical Physics, 61 (3), 033405-1-033405-4.

https://scholar.uwindsor.ca/physicspub/12

This Article is brought to you for free and open access by the Department of Physics at Scholarship at UWindsor. It has been accepted for inclusion in Physics Publications by an authorized administrator of Scholarship at UWindsor. For more information, please contact scholarship@uwindsor.ca. 


\title{
Photodetachment in combined static and dynamic electric fields
}

\author{
Chitra Rangan and A. R. P. Rau \\ Department of Physics and Astronomy, Louisiana State University, Baton Rouge, Louisiana 70803-4001
}

(Received 19 November 1998; published 14 February 2000)

\begin{abstract}
Through an exact solution of the time-dependent Schrödinger equation for an electron in a static electric field plus the time-dependent electric field of the detaching radiation, the photodetachment cross section of $\mathrm{H}^{-}$ is calculated. Careful attention is paid to ensuring proper limiting behavior as the frequency of the timedependent field goes to zero. We do not find observable effects of a cross term between the two fields on the detachment cross section. Our results point to possible gauge dependence and other difficulties of $S$-matrix formulations of multiphoton detachment and ionization.
\end{abstract}

PACS number(s): 32.60.+i, 32.80.Gc

Following a detailed experimental study [1] of the photodetachment of $\mathrm{H}^{-}$just above threshold in the presence of a strong electric field $(\sim 100 \mathrm{kV} / \mathrm{cm})$, theoretical analyses [2-4] accounted for the principal observed effects: a finite cross section at the zero-field detachment threshold of 0.75 $\mathrm{eV}$, an exponential falloff of the cross section for lower photon energies due to detachment aided by tunneling through the static field's potential, and oscillations in the cross section about the zero-field value for energies above $0.75 \mathrm{eV}$. This last may be viewed either as the effect of the sloping static field potential on the outgoing $p$ wave ("Airy-function oscillations") or as the interference between two pathways for the escaping electron, one directly into the escape direction and the other after reflection of an oppositely moving wave from the static field barrier. Such simple, and analytical, treatments of the effect of a static field on a free outgoing $p$ electron, with neglect of final-state interactions between it and the parent $\mathrm{H}$ atom left behind, sufficed to give a complete and detailed accounting of the observed data [2,3]. Treatment of such final-state interactions has also been carried out subsequently [5].

With the advent of intense lasers, there has also been interest in multiphoton detachment and in nonperturbative phenomena due to the dynamic electric field of the detaching laser [6,7]. In particular, Gao and Starace [6] reinvestigated the problem through an exact solution for the outgoing electron in combined static and dynamic electric fields. When applied to $\mathrm{H}^{-}$, they claimed that a cross term between the two fields leads to somewhat different results from previous studies even in the weak-laser-field limit, the cross section near the zero-static-field detachment threshold being lowered. This is the question we address here through an alternative derivation of this exact solution that pays careful attention to its proper limiting behaviors. Our results do not support the claims of lowered cross sections arising from a cross term between the static and dynamic fields. On the other hand, our analysis suggests a more general caution that may apply to $S$-matrix formulations because results seem to depend on the choice of gauge for the electromagnetic potentials.

As in Ref. [6], and adopting the same notation, we consider a uniform static electric field $\vec{E}_{s}=E_{s} \hat{\mathbf{z}}$ and the timedependent electric field of the laser (effects of its magnetic field are as usual neglected as smaller) to give a total field

$$
\vec{E}(t)=\vec{E}_{s}+\vec{E}_{0} \sin \omega t=E_{s} \hat{\mathbf{z}}+\left(E_{0 x} \hat{\mathbf{x}}+E_{0 y} \hat{\mathbf{y}}+E_{0 z} \hat{\mathbf{z}}\right) \sin \omega t .
$$

Describing the outgoing electron as moving in such a timedependent field, and neglecting any residual interactions with the $\mathrm{H}$ atom left behind, the Schrödinger equation

$$
i \Psi(\vec{r}, t)=\left[\vec{p}^{2} / 2+\vec{E}(t) \cdot \vec{r}\right] \Psi(\vec{r}, t)
$$

can be solved through separation in Cartesian coordinates. Throughout, an overdot will denote differentiation with respect to $t$ and we set $\hbar=m=e=1$. Whereas Ref. [6] did so through a passage to momentum space, we develop our solutions in coordinate space through a technique of solving such time-dependent equations by operator algebra [8]. By working in coordinate space and directly with the electric fields themselves, we avoid questions that arise in the momentum space formalism, particularly the gauge choice for the vector potential $\vec{A}$.

For a general time-dependent equation,

$$
i \dot{\phi}(t)=[\dot{\mu}(t) A+\dot{\nu}(t) B] \phi(t),
$$

where $A$ and $B$ are possibly noncommuting operators not themselves explicitly dependent on time, the general solution can be developed in terms of an evolution operator, $U(t, 0)$, which is of the form of a product of exponentials, each involving $A, B$, and successive commutators of them, along with time-dependent functions $\mu(t), \nu(t), \lambda(t), \delta(t), \ldots$. which obey first-order classical differential equations [8]. Each Cartesian component in Eq. (2) involves only the operators $p^{2}$ and the linear coordinate so that apart from their commutator proportional to $p$, no further operators appear. The resulting solutions involve four exponential factors.

For the $x$ and $y$ components, these solutions have been previously recorded [8] and are

$$
\begin{aligned}
\Psi(x, t)= & \exp \left[-i\left(E_{0 x}^{2} / \omega^{3}\right)\left(\frac{1}{8} \sin 2 \omega t-\sin \omega t+3 \omega t / 4\right)\right] \\
& \times \exp \left[-i E_{0 x}(1-\cos \omega t) / \omega\right] \\
& \times \exp \left[-i\left(E_{0 x} k_{x} / \omega^{2}\right)(\sin \omega t-\omega t)\right] \\
& \times \exp \left[i k_{x} x-i k_{x}^{2} t / 2\right]
\end{aligned}
$$


with an exactly similar expression in $y$.

The solution in the $z$ coordinate can be similarly derived through the procedure in Ref. [8] to give

$$
\begin{aligned}
\Psi(z, t)= & \exp \left[-i\left(E_{0 z}^{2} / \omega^{3}\right)\left(\frac{1}{8} \sin 2 \omega t-\sin \omega t+3 \omega t / 4\right)\right. \\
& \left.+i\left(E_{0 z} E_{s} / \omega^{3}\right)\left(\cos \omega t-1+\omega^{2} t^{2} / 2\right)\right] \\
& \times \exp \left[-i E_{0 z} z(1-\cos \omega t) / \omega\right] \exp \left[-i\left(E_{0 z} p_{z} / \omega^{2}\right)\right. \\
& \times(\sin \omega t-\omega t)] \exp \left(-i \varepsilon_{z} t\right) \phi_{A}(z),
\end{aligned}
$$

where $\varepsilon_{z}$ is the eigenvalue and $\phi_{A}(z)$ the Airy eigenfunction [9] satisfying

$$
\left(\frac{p_{z}^{2}}{2}+E_{s} z\right) \phi_{A}(z)=\varepsilon_{z} \phi_{A}(z) .
$$

These results in Eqs. (4) and (5) parallel exactly the similar expressions in Ref. [6] but with additional phases that are central to our discussion. An important difference is that we have paid careful attention to the boundary condition at $t$ $=0$, that Eq. (4) reduce to plane waves in $x$ and $y$ with no extraneous phase factors and Eq. (5) to the Airy stationary state with again each of the three exponentials in the top two lines of this equation reducing exactly to unity. Construction through the evolution operator ensures this reduction [8]. In contrast, Ref. [6]'s solutions retain at $t=0$, when the field $\vec{E}_{0} \sin \omega t$ vanishes, redundant phase factors because of their choice of the vector potential $\vec{A}=\left(c \vec{E}_{0} / \omega\right) \cos \omega t$ to describe this electric field while working in momentum space. Indeed, were we to modify their results by replacing $\cos \omega t$ above by (cos $\omega t-1)$ in $\vec{A}$, which still describes the same electric field, the two results would become identical. As we will see, this seemingly slight difference in the choice of a gauge has important consequences. In our formalism, however, we work directly with the electric field in Eq. (1).

The appearance in Eqs. (4) and (5) of the characteristic combinations $(\sin \omega t-\omega t)$ and $\left(\cos \omega t-1+\omega^{2} t^{2} / 2\right)$, in place of the trigonometric functions alone as in Ref. [6], has another profound consequence. Our solutions remain well behaved in the limit $\omega \rightarrow 0$ (which is closely related to $t \rightarrow 0$ because of the combination $\omega t$ ) whereas some of the similar terms in Ref. [6] and, in particular, the cross term involving $E_{0 z} E_{s}$ in Eq. (5), blow up in this limit. This is a persistent problem in the literature on intense dynamic fields [10], that several results seem not to admit passage to the static field limit as one would expect of them. It is worth emphasizing that within the momentum space formulation as in Ref. [6], different choices of $\vec{A}$, differing only in a constant which does not change the electric field, lead to different wave functions. In particular, these functions can differ drastically in the $\omega \rightarrow 0$ limit so that particular care may be necessary for considerations of the static field limit. We also note that these gauge questions have to do with alternative wave functions for alternative vector potentials, not whether the dipole matrix element for photoabsorption uses length, velocity, or acceleration forms.
To calculate the photodetachment of $\mathrm{H}^{-}$, we follow Ref. [6] in using Eqs. (4) and (5) to describe the final-state wave function along with a simple, much used "one-electron", representation of the ground state of $\mathrm{H}^{-}[2,3,6,11]$,

$$
\Psi_{i}(\vec{r}, t)=(B / r) \exp (-k r) \exp \left(-i \varepsilon_{i} t\right)
$$

where $k=\left(-2 \varepsilon_{i}\right)^{1 / 2}, \varepsilon_{i}=-0.75 \mathrm{eV}$, being the energy of this ground state, and $B$ is a normalization parameter, equal to 0.31552 in atomic units $[3,6]$. This wave function has long [11] proved very successful in describing photodetachment for the energy range of interest and has been employed in all the past work that we compare with. The $S$-matrix element is given as in Eq. (27) of Ref. [6] by

$$
\begin{aligned}
S_{f i}= & (2 \pi)^{-1 / 2} i B \int_{-\infty}^{\infty} d t \int d \vec{r} \Psi^{*}(x, t) \Psi^{*}(y, t) \Psi^{*}(z, t) \\
& \times \exp \left(-i \varepsilon_{i} t\right)
\end{aligned}
$$

with the wave functions drawn from Eqs. (4) and (5).

Examining next the weak-laser-field limit, we expand the factors $\Psi^{*}$ to first order in $E_{0}$ and retain terms proportional to it to get

$$
\begin{aligned}
S_{f i}= & \frac{i 2^{2 / 3} B E_{0 z} E_{s}^{1 / 6}}{\omega^{2}} \\
& \times\left\{\operatorname{Ai}^{\prime}(-\xi) \int_{-\infty}^{\infty} d t(\sin \omega t-\omega t) \exp \left[i\left(\varepsilon_{f}-\varepsilon_{i}\right) t\right]\right. \\
& -i \frac{\left(E_{s}^{2} / 2\right)^{1 / 3}}{\omega} \operatorname{Ai}(-\xi) \int_{-\infty}^{\infty} d t \\
& \left.\times\left(\cos \omega t-1+\frac{1}{2} \omega^{2} t^{2}\right) \exp \left[i\left(\varepsilon_{f}-\varepsilon_{i}\right) t\right]\right\}
\end{aligned}
$$

where $\varepsilon_{f}=\frac{1}{2}\left(k_{x}^{2}+k_{y}^{2}\right)+\varepsilon_{z}$ and we have defined a dimensionless energy $\xi=\varepsilon_{z}\left(2 / E_{s}^{2}\right)^{1 / 3}$. The derivative in the first term, denoted by a prime, is with respect to $\xi$, and arises from the $E_{0 z} p_{z}$ operator in Eq. (5).

The above result in Eq. (9) parallels exactly the one in Ref. [6], again with the replacement of $\sin \omega t$ and $\cos \omega t$ by the forms that vanish up through terms of order $\omega^{2} t^{2}$. Before turning to the time integrations, the structure of Eq. (9) and the origin of its second term in the curly brackets already point to problems with the claim in Ref. [6] that this is a new contribution not present in earlier treatments. Whereas the first term in Eq. (9) arises from the expansion of the $E_{0 z} p_{z}$ in Eq. (5), thus carrying the dipole operator that leads to the transition element $\left\langle\Psi\left|E_{0 z} p_{z}\right| \Psi_{i}\right\rangle$, the second term has its origins in the cross term $E_{0 z} E_{s}$ of Eq. (5). But this is purely a phase with no involvement of atomic operators, and therefore incapable of causing transitions.

Further confirmation of this conclusion that there is no cross term proportional to $E_{s} E_{0 z}$ is provided by carrying out the time integrations. In Ref. [6], these integrations were immediate, upon combining the $\exp (-i \omega t)$ piece of the sine 


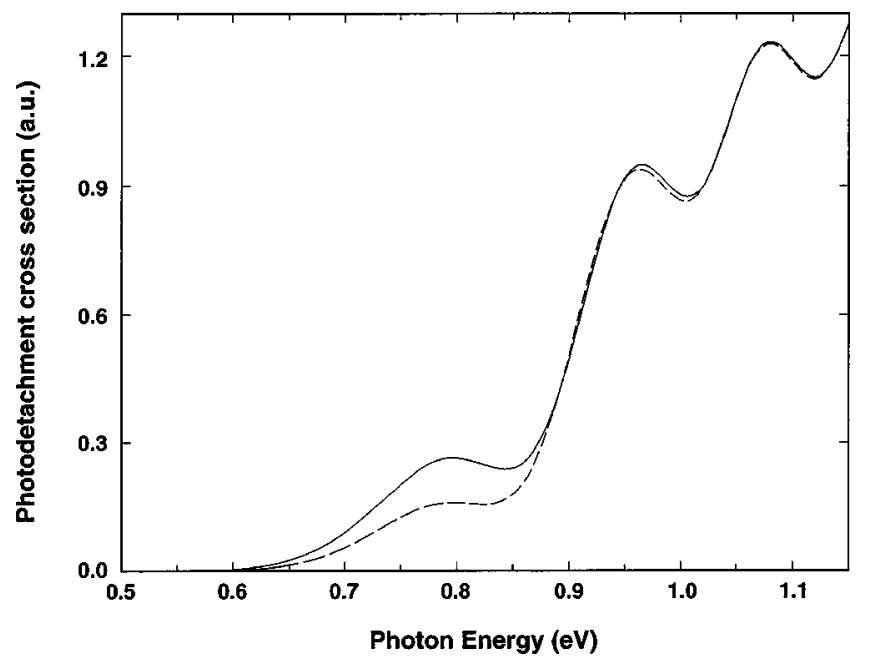

FIG. 1. Photodetachment of $\mathrm{H}^{-}$in combined static $(1 \mathrm{MV} / \mathrm{cm})$ and detaching laser's electric fields. - , our results; --- , Ref. [6]. Earlier calculations of Refs. [2] and [3] are essentially indistinguishable from the solid line.

and cosine with the other exponential in the integrand to give, upon integration, $2 \pi \delta\left(\varepsilon_{f}-\varepsilon_{i}-\omega\right)$. With the transition probability $W_{f i}$ defined as

$$
\lim _{t \rightarrow \infty} \frac{\left|S_{f i}\right|^{2}}{t}=2 \pi W_{f i} \delta\left(\varepsilon_{f}-\varepsilon_{i}-\omega\right),
$$

the cross section

$$
\sigma=\frac{8 \pi \omega}{c E_{0}^{2}} \int W_{f i} d k_{x} d k_{y} d \varepsilon_{z}
$$

was then evaluated.

We now handle these operations by evaluating the integrals in Eq. (9) between limits $-T$ and $T$ analytically and computing $\sigma$, finally taking the $T \rightarrow \infty$ limit numerically. In the first part of this process, the analytical integration from $-T$ to $T$, we retain only the contributions that lead to $\delta\left(\varepsilon_{f}\right.$ $\left.-\varepsilon_{i}-\omega\right)$ in the limit $T \rightarrow \infty$ as the only ones that correspond to absorption of a single photon. As $T$ increases beyond a few atomic units, our calculated photodetachment cross section converges rapidly. The second term in Eq. (9) does not contribute in accordance with our discussion above and, as illustrated in Fig. 1, our results reproduce exactly those of earlier work $[2,3]$ even for static field strengths exceeding 1 $\mathrm{MV} / \mathrm{cm}$. All these results coincide as shown by the solid curve. Only if both terms in the curly bracket in Eq. (9) are retained, while simultaneously dropping the terms in $-\omega t$ and $-1+\frac{1}{2} \omega^{2} t^{2}$, do we recover the results of Ref. [6] as shown by the dashed curve. We conclude, therefore, that a proper treatment leads to no reduction in the cross section around the zero-static-field threshold as claimed in Ref. [6]. The handling of time integrations with the $-\omega t$ and -1 $+\frac{1}{2} \omega^{2} t^{2}$ terms would also confront a calculation such as the one in Ref. [6] had this alternative gauge been used for $\vec{A}$.
The $S$-matrix integrations in Eq. (9) would then not simply reduce to $\delta$ functions but also involve the derivatives that we have encountered.

In view of the discrepancy with the previous results in Ref. [6], we offer the following discussion. In the spirit of the $S$-matrix formulation, the electric field in Eq. (1) is assumed to be switched on starting at $t=-\infty$ and switched off at $t=\infty$ adiabatically, although this is not explicitly implemented in carrying out the time integrations, just as in Ref. [6]. Therefore, our calculations follow exactly the same procedure as did Ref. [6], except that our final state wave functions in Eqs. (4) and (5) differ from those in Ref. [6] as pointed out above. We were led to these additional terms involving $-\omega t$ and $-1+\frac{1}{2} \omega^{2} t^{2}$ by our emphasis on the proper limiting behavior as $t \rightarrow 0$ or $\omega \rightarrow 0$. Therefore, it may be argued that Ref. [6] and this paper deal with different problems, differing in when the electric fields are turned on. But, as we have pointed out, these additional terms may also be viewed as arising from the two different gauge choices for $\vec{A}$, namely, $\left(c \vec{E}_{0} / \omega\right)(\cos \omega t-1)$, with or without that -1 . As shown in Fig. 1, the difference between the dashed and solid lines can be attributed entirely to this difference, which in itself poses the question of gauge invariance of the cross sections presented in Ref. [6].

Some of these questions of the switching on and off of the electric fields can be settled by developing explicit solutions of Eqs. (1) and (2) with some specific form of $E(t)$ that vanishes smoothly as $|t| \rightarrow \infty$, although this might require an additional numerical integration, that in $t$, for the calculation. We also disagree with Ref. [6] in other regards. Most importantly, it seems to us that notwithstanding any multiple order of interactions with the static field $E_{s}$, as interpreted in the $S$-matrix formalism, one-photon absorption in the limit of weak laser fields must involve an amplitude proportional to $E_{0}$, along with the matrix element of a dipole operator, whether $\vec{r}$ or $\vec{p}$, and a corresponding energy-conserving delta function $\delta\left(\varepsilon_{f}-\varepsilon_{i}-\omega\right)$. Therefore, one-photon transitions can be attributed only to terms that have such a structure upon expanding exponentials in Eqs. (4) and (5) to first order in $E_{0}$. The $E_{0 z} E_{s}$ term of Ref. [6] does not satisfy these requirements. Likewise, in a related argument that may help to clarify the points of disagreement, were we to seek twophoton transitions by expanding the exponentials to order $E_{0}^{2}$, we would not expect any contribution from the first terms involving $E_{0}^{2} \sin 2 \omega t$ in Eqs. (4) and (5) because they contain no atomic operators. In this, we would differ from Ref. [6] and other such treatments, whose Floquet expansions get a contribution from these terms, the so-called "ponderomotive potential" then appearing in their resulting energy-conserving $\delta$ function for such two- (or multiple-) photon transitions. Since this ponderomotive potential depends on the continuous variable $E_{0}^{2} / \omega^{2}$, it need not be an integer multiple of $\omega$, placing it in conflict with the photon picture of multiphoton absorption. We, on the other hand, would face no such conflict (see also Ref. [12]).

Finally, after completion of our work, we saw a recent paper [13] on the one- and two-photon photodetachment of $\mathrm{H}^{-}$in combined static and dynamic fields, taking into ac- 
count final-state interactions of the electron with the residual $\mathrm{H}$ atom. As already stated, our study was not concerned with this interaction. But we note that Ref. [13]'s treatment of such an interaction leads also to contributions similar in structure to the "cross term" in $E_{0 z} E_{s}$, namely, to a term proportional to $\mathrm{Ai}(-\xi)$ in Eq. (9); see Eq. (72) of [13]. In disentangling the two effects, of the cross term and the electron-atom final-state interaction, and in coming to the conclusion that the latter is small, Ref. [13] has compared with the previously calculated effects of the cross term in Ref. [6]. In view of our questioning of any depression of the cross section around the zero-field detachment threshold due to the cross term, the conclusion of Ref. [13] on the unimportance of the electron-atom final-state interaction will have to be revisited.

We have also seen a recent paper [14] on $\mathrm{H}^{-}$photodetachment in a static electric field and a pulsed laser field. The authors consider quantum and semiclassical approaches different from both ours and Ref. [6]. These authors also note in a footnote to their Eq. (6) that only a term in the derivative of, but not in $\operatorname{Ai}(-\xi)$ itself, contributes, in agreement with our conclusions.

We acknowledge useful discussions with Dr. A. F. Starace and Dr. K. Unnikrishnan.
[1] H. C. Bryant, A. Mohagheghi, J. E. Stewart, J. B. Donahue, C. R. Quick, R. A. Reeder, V. Yuan, C. R. Hummer, W. W. Smith, S. Cohen, W. P. Reinhardt, and L. Overman, Phys. Rev. Lett. 58, 2412 (1987).

[2] A. R. P. Rau and H. Y. Wong, Phys. Rev. A 37, 632 (1988); 38, 1660 (1988); H. Y. Wong, A. R. P. Rau, and C. H. Greene, ibid. 37, 2393 (1988).

[3] M. L. Du and J. B. Delos, Phys. Rev. A 38, 5609 (1988); M. L. Du, ibid. 40, 4983 (1989).

[4] C. H. Greene and N. Rouze, Z. Phys. D: At., Mol. Clusters 9, 219 (1988); V. D. Kondratovich and V. N. Ostrovskii, J. Phys. B 23, 21 (1990); I. I. Fabrikant, ibid. 23, 1139 (1990); Zh. Eksp. Teor. Fiz. 79, 2070 (1980) [Sov. Phys. JETP 52, 1045 (1980)]; 83, 1675 (1982) [56, 967 (1983)].

[5] I. I. Fabrikant, Phys. Rev. A 40, 2373 (1989); C. A. Nicolaides and Th. Mercouris, Chem. Phys. Lett. 159, 45 (1989); V. Z. Slonim and F. I. Dalidchik, Zh. Eksp. Teor. Fiz. 71, 2057 (1976) [Sov. Phys. JETP 44, 1081 (1976)].
[6] B. Gao and A. F. Starace, Phys. Rev. A 42, 5580 (1990).

[7] I. N. Artyunyan and G. A. Askar'yan, Pis'ma Zh. Eksp. Teor. Fiz. 12, 378 (1970) [JETP Lett. 12, 259 (1970)]; A. I. Nikishov, Zh. Eksp. Teor. Fiz. 62, 562 (1972) [Sov. Phys. JETP 35, 298 (1972)]; V. L. Manakov and A. G. Fainshtein, ibid. 79, 751 (1980) [ibid. 52, 382 (1980)].

[8] A. R. P. Rau and K. Unnikrishnan, Phys. Lett. A 222, 304 (1996).

[9] Handbook of Mathematical Functions, edited by M. Abramowitz and I. A. Stegun (Dover, New York, 1972), Sec. 10.4 .

[10] P. W. Milonni and Bala Sundaram, Prog. Opt. 31, 1 (1993).

[11] T. Ohmura and H. Ohmura, Phys. Rev. 118, 154 (1960).

[12] A. R. P. Rau, Phys. Rev. A 54, 717 (1996).

[13] M. Q. Bao, I. I. Fabrikant, and A. F. Starace, Phys. Rev. A 58, 411 (1998).

[14] Y. Zhao, M. Du, and J. Mao, J. Phys. B 32, 1409 (1999). 